\title{
NAMPT (VISFATIN) INFLUENCE ON PROLIFERATIVE ACTIVITY OF NORMAL RAT ADRENOCORTICAL CELLS AND HUMAN ADRENAL CORTICOCARCINOMA NCI-H295R CELLS
}

Piotr Celichowski ${ }^{1 *}$, Karol Jopek ${ }^{1}$, Marta Szyszka $^{1}$, Paulina Milecka ${ }^{1}$, Ludwik K. Malendowicz ${ }^{1}$, Marianna Tyczewska ${ }^{1,2}$, Marcin Ruciński ${ }^{1}$

\begin{abstract}
Nampt (Nicotinamidephosphoribosyltransferase - also known as visfatin/PBEF) is the enzyme that regulates the NAD+ level, therefore influencing many metabolic pathways within the cells. As circulating cytokine, extracellular Nampt (eNampt) exerts pro-inflammatory, pro-chemotactic, pro-angiogenic and insulin-like effects; however the mechanism of eNampt action is still unclear.Earlier studies have shown that eNampt exerts a stimulating effect on the proliferation of many cancer cell lines. However, the effect of this cytokine on cell proliferation in primary culture is little known. Therefore, the aim of the study was to analyse the influence of eNampt on the proliferation of rat adrenocortical cells in primary culture and to investigate similar influence of eNampt on the line H295R of human adrenal corticocarcinoma cells. Proliferation of the examined cells was assessed using the RTCA (Real Time Cell Analyzer) method. The obtained results indicate that eNampt stimulates the proliferation of H295R cells, but does not change the proliferation of cultured rat adrenocortical cells. In primary culture of rat adrenocortical cells, Fk866 (specific Nampt inhibitor) does not modify the rate of proliferation of tested cells. In H295R cells the addition of Fk866 alone inhibits proliferative activity and stimulates apoptosis. Fk866 also inhibits the stimulating effect of eNampt on H295R cell proliferation.
\end{abstract}

Running title: Nampt influence on proliferative activity of adrenocortical cells

Keywords: Nampt, Visfatin, H295R, adrenals, adrenocortical, proliferation

\footnotetext{
${ }^{1}$ Department of Histology and Embryology, Poznan University of Medical Sciences, Poznań, Poland

${ }^{2}$ Department of Anatomy and Histology, University of Zielona Góra, Zielona Góra, Poland

* Correspondence: p.celichowski@gmail.com

Full list of author information is available at the end of article
} 


\section{Introduction}

Nampt (Nicotinamidephosphoribosyltransferase - also known as visfatin/PBEF) is an enzyme regulating NAD+ level in cells [1]. The NAD synthesis originates from 4 main substrates, such as tryptophan (Trp), nicotinamide (NAM), nicotinic acid (NA), and nicotinamideriboside (NR), but in mammals the main substrate $(\sim 90 \%)$ is nicotinamide (NAM) [2, 3]. Moreover, the fact that intracellular NAD levels increase with Nampt gene overexpression in multiple, diverse cell types suggests that Nampt expression is sufficient to induce NAD biosynthesis [4-10]. NAD+ is used as a substrate by poly-ADP-ribose polymerases, sirtuins, and ADP-ribosyltransferases, which respectively play important roles in DNA damage repair, deacetylation, and generation of second messengers required for intracellular calcium release $[11,12]$.

There is already much evidence of the strong effect of Nampt on metabolism. Many of carcinogenesis factors, such as SirT1, CtBP and PARP-1 are induced by its action, indicating Nampt as one of the most important factors of cancer malignances [13] Moreover, Nampt gene expression was found to be higher in tumor cells than normal cells [11,13-20].

As a circulating cytokine, eNampt is mainly secreted by adipose tissues, with macrophages, hepatocytes, chondrocytes, leucocytes, inflamed endothelial cells, human peripheral blood lymphocytes, CLL lymphocytes, gastric cancer cells and even antigen presenting cells (APCs) being possible sources of eNampt [11]. eNampt has been reported to has pro-inflammatory, prochemotactic, proangiogenic and insulin-like effects [21, 22].

As known, Nampt plays a central role in the systemic regulation of NAD biosynthesis in cells, hence it can also regulate their proliferation. In the available literature there are many publications that this cytokine exerts dose-dependent stimulating effect on proliferative activity, mainly of cancer cells [18, 23-28]. This stimulation occurred probably through AKT/PI3K, ERK/MAPK and GSK-3 $\beta$ proteins $[23,25,26,28]$.

Only a few publications indicate that Nampt regulates the function of steroidogenic cells, as well as the function of hypothalamo-pituitary-adrenal axis of the rat [29-33]. However, the role of Nampt in the regulation of proliferation of steroidogenic cells is not known. Therefore, the aim of the presented experiments was to investigate Nampt's influence on proliferative activity of normal rat adrenocortical cells and human adrenal corticocarcinoma NCIH295R cells.

\section{Materials and methods Animals and reagents}

Wistar rats were obtained from the Laboratory Animal Breeding Centre of the Department of Toxicology, Poznan University of Medical Sciences (Poznan, Poland). The animals were maintained un- der standard light conditions (14:10 h light/dark cycle, illumination onset at 6.00 a.m.) at $23^{\circ} \mathrm{C}, 50-60 \%$ air humidity, 8-10 air changes per hour (mechanical, via HEPA filters) with free access to standard pellets and tap water. The study protocol was approved by the independent Local Ethics Committee for Animal Studies in Poznań (protocol no. 75/2016). Recombinant human Nampt protein was purchased from BioVendor R\&D Products (Brno, Czechia) and the specific Nampt inhibitor Fk866 was purchased from ApexBio Technology (Houston, TX, USA). The ACTH (Cortrosyn $囚$ ) was purchased from Organon (Merck \& Co, USA).

\section{Primary culture of rat adrenocortical cells}

The method used for culturing rat adrenocortical cells has been described previously [33-36]. Briefly, adrenal glands were obtained from 35 60-80 g, 21-day-old, male rats. Glands were immediately transferred into a vessel with culture medium (DMEM/F12 without phenol red), mechanically chopped and digested with collagenase type I (1 $\mathrm{mg} / \mathrm{ml}$ in DMEM/F12 medium; Sigma Aldrich; Merck KGaA) in a water bath at $37^{\circ} \mathrm{C}$ for $30 \mathrm{~min}$. The suspension was further mechanically disintegrated using a glass pipette and poured through a nylon filter into a test tube, followed by centrifugation (200 $\mathrm{x} g$ for $10 \mathrm{~min}$ at room temperature). The collected cells were subsequently suspended in DMEM/F12 (11039-021, ThermoFisher Scientific, Waltham, MA, USA)with 10\% FBS (F7524, Sigma-Aldrich; Merck KGaA) and Antibiotic Antimycotic Solution (A5955, Sigma-Aldrich; Merck KGaA) and plated on culture plates.

\section{Human adrenal corticocarcinoma NCI-H295R cell culture}

Human H295R adrenocortical cells were obtained from the American Type Culture Collection (Manassas, VA, USA) and cultured in H295R complete media containing DMEM/F12 (11039-021, ThermoFisher Scientific, Waltham, MA, USA) supplemented with $2.5 \% \mathrm{Nu}$-Serum $^{\mathrm{TM}}$ (355500, Corning, NY, USA) and ITS (insulin transferrin selenium) (354350, Corning, NY, USA). The cells were cultured in standard $37^{\circ} \mathrm{C}$ and $5 \% \mathrm{CO}_{2}$ conditions.

\section{Determination of the proliferation rate of cultured cells}

Proliferation rate of cultured cells was estimated by means of the xCelligence Real-Time Cell Analyzer (RTCA) DP (dual purpose) (Roche). The system measures real-time changes in electrical impedance across microelectrodes integrated into the bottom of tissue culture E-Plates. The impedance measurement provides quantitative information about the biological status of the cells, including cell number, viability and morphology. Studies were performed on cells prepared as described above. The measured 
number of cells was displayed as cell index. Cell Index is a unitless parameter proportional to the number of cells measured by impedance of electron flow caused by adherent cells. Fifty microliters of cell culture medium was added to each well for the impedance background measurement. After adding the cells and/or drugs, the final volume was $200 \mu \mathrm{L}$. The E-Plates were incubated at $37^{\circ} \mathrm{C}$ with $5 \% \mathrm{CO}_{2}$ and monitored on the RTCA system at 15-minute time intervals for $80 \mathrm{~h}$ (rat adrenocortical cells) or 150-200 h (H295R cells) with or without treatment.

\section{Treatment of cultured cells}

From 48 h onwards, proliferating cells were exposed to tested substances (eNampt, Fk866, ACTH) individually or in combination, at different concentrations, as shown in Figures 1-3. All test substances were added once in $48 \mathrm{~h}$ of culture. The concentrations of administrated eNampt were chosen based on the physiological eNampt serum levels in humans, which were reported around $1-4 \mathrm{ng} / \mathrm{ml}$ $\left(\sim 10^{-8} \mathrm{M}\right)$ [37-39]. The serum eNampt concentration of the rat is similar to that of humans [40].

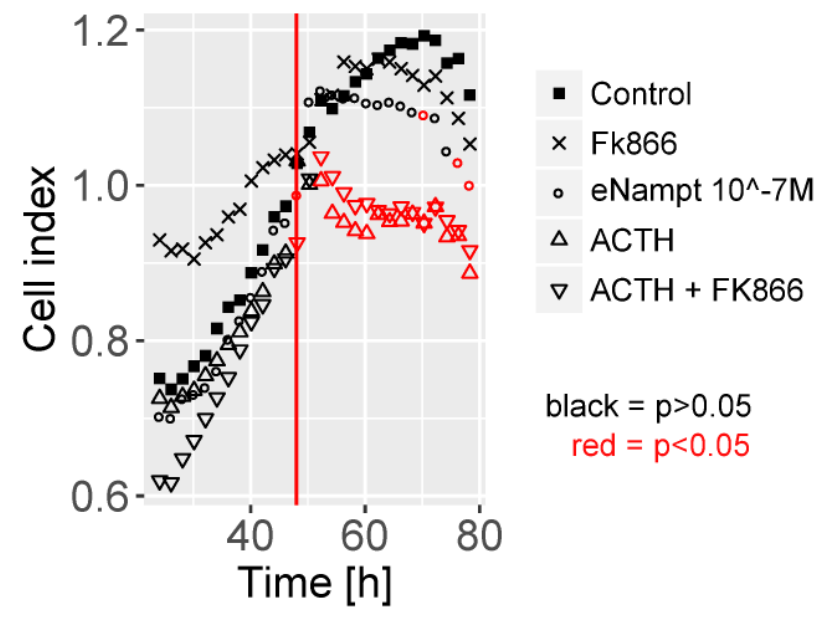

Figure 1 Primary culture of rat adrenocortical cells. RTCA (Real Time Cell Analyzer) delivered cell index. Cells were cultured for $80 \mathrm{~h}$ in the presence of eNampt $\left(1 \times 10^{-7} \mathrm{M}\right)$, Fk866 $\left(1 \times 10^{-7} \mathrm{M}\right)$ and ACTH $\left(1 \times 10^{-7} \mathrm{M}\right)$ with or without Fk866. Normalized RTCA chart plot (cell index - recorded every $15 \mathrm{~min}$ ) in which points are marked every 45 minutes. $\mathrm{n}=4$ per group. The red vertical line represents the time of data normalization (in which the number of cells has been set at 1) and the administration of tested substances. Statistically significant differences in relation to the control group (by using Kruskal-Wallis one-way analysis of variance (ANOVA) followed by Wilcoxon-Mann-Whitney post-hoc test) are indicated by a red colour, $\mathrm{p}<0.05$. Black - no differences. eNampt, extracellular nicotinamide phosphoribosyltransferase; ACTH, adrenocorticotropic hormone; Fk866, specific Nampt inhibitor

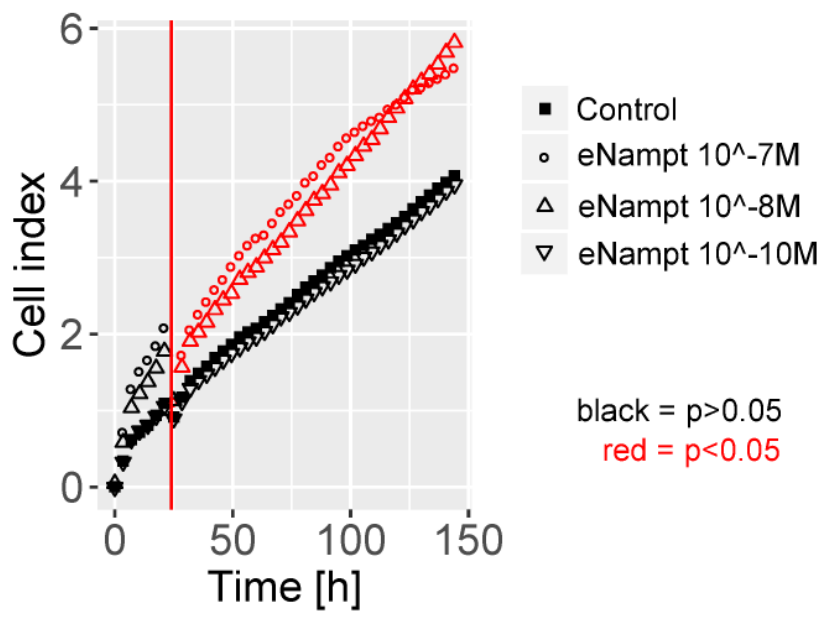

Figure 2 Culture of H295R human adrenocortical cells. RTCA (Real Time Cell Analyzer) delivered cell index. Cells were cultured for $150 \mathrm{~h}$ in the presence of eNampt $\left(1 \times 10^{-7} \mathrm{M} ; 1 \times 10^{-8} \mathrm{M} ; 1 \times 10^{-10} \mathrm{M}\right)$. Normalized RTCA chart plot (cell index - recorded every $15 \mathrm{~min}$ ) in which points are marked every 45 minutes. $n=5-8$ per group. The red vertical line represents the time of data normalization (in which the number of cells has been set at 1) and the administration of eNampt. Statistically significant differences in relation to the control group (by using Kruskal-Wallis one-way analysis of variance (ANOVA) followed by Wilcoxon-Mann-Whitney post-hoc test) are indicated by a red colour, $\mathrm{p}<0.05$. Black no differences. eNampt, extracellular nicotinamide phosphoribosyltransferase 


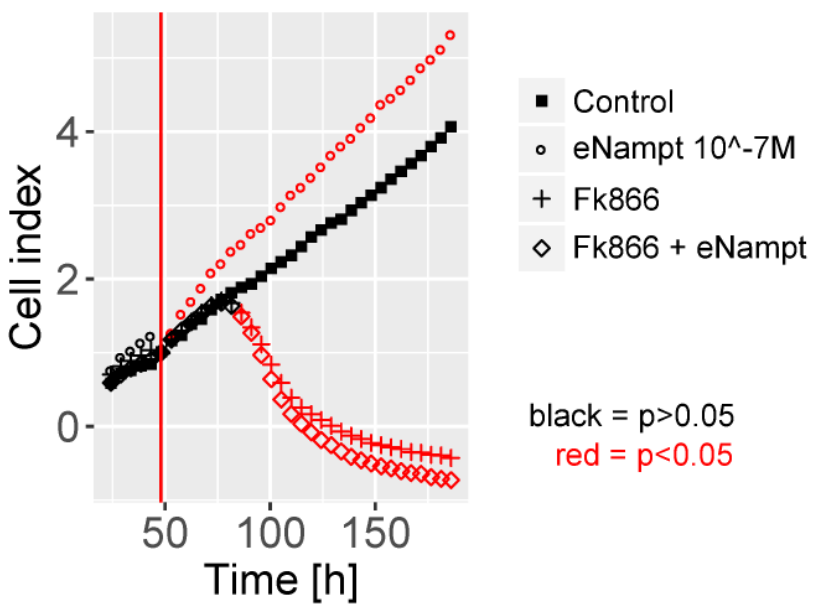

Figure 3 Culture of H295R human adrenocortical cells. RTCA (Real Time Cell Analyzer) delivered cell index. Cells were cultured for $200 \mathrm{~h}$. Cells were exposed to Fk866 $\left(1 \times 10^{-7} \mathrm{M}\right)$ with or without eNampt $\left(1 \times 10^{-7} \mathrm{M}\right)$. Normalized RTCA chart plot (cell index - recorded every $15 \mathrm{~min}$ ) in which points are marked every 45 minutes. $\mathrm{n}=5-8$ per group. The red vertical line represents the time of data normalization (in which the number of cells has been set at 1) and the administration of tested substances. Statistically significant differences in relation to the control group (by using Kruskal-Wallis one-way analysis of variance (ANOVA) followed by Wilcoxon-Mann-Whitney post-hoc test) are indicated by a red colour, $\mathrm{p}<0.05$. Black - no differences. eNampt, extracellular nicotinamide phosphoribosyltransferase; Fk866, specific Nampt inhibitor

\section{Statistical analysis}

The curves of the cell index were normalized by means of software of RTCP system. The diagrams show the means of repeated experiments (for rat 4 repetitions; for H295R cells5-8 repetitions). For multiple comparisons, statistical analysis of the data was performed by using Kruskal-Wallis one-way analysis of variance (ANOVA) followed by Wilcoxon-Mann-Whitney post-hoc test between all measurements. Calculations were performed using R x64 3.4.1 software.

\section{Ethical approval:}

The research related to animals use has been complied with all the relevant national regulations and institutional policies policies for the care and use of animals. Bioethical Committee approval no. 75/2016.

\section{Results}

As can be seen from fig. 1, neither the addition of eNampt $\left(1 \times 10^{-7} \mathrm{M}\right)$, nor the addition of Fk866 $\left(1 \times 10^{-7} \mathrm{M}\right)$, a specific Nampt inhibitor, change the proliferation of rat adrenocortical cells in the primary culture. In these conditions, the addition of ACTH (1x10-7 M) alone or with FK866 inhibits the proliferation of tested cells. In the case of human adrenal corticocarcinoma H295Rcells, eNampt in concentrations of $1 \times 10^{-7} \mathrm{M}$ and $1 \times 10^{-8} \mathrm{M}$ significantly stimulate proliferative activity of the studied cells (fig. 2). On the other hand, eNampt at concentrations of $1 \times 10^{-10} \mathrm{M}$ does not influence the proliferation of these cells. As shown in fig. 3, Fk866 (specific Nampt inhibitor) alone in the concentration of $1 \times 10^{-7} \mathrm{M}$ inhibits proliferation of H295R cells and inhibits stimulating effect of eNampt (1x10-7 M) on proliferation of these cells.

\section{Discussion}

Our research has revealed very interesting results regarding Nampt's role in the regulation of adrenocortical cell proliferation, both normal and neoplastic. In our experiments we have observed that eNampt exerts a stimulating effect on the proliferation of H295R human adrenal corticocarcinoma cells, but does not show such an effect on normal rat adrenocortical cells in primary culture. In addition, Fk866, a specific Nampt inhibitor, does not affect the proliferation rate of rat adrenocortical cells under the experimental conditions applied, while this compound strongly inhibits mitotic activity of H295R cells.

Numerous previous studies on established cell lines have shown that eNampt exerts a stimulating effect on the rate of proliferation of different cancer cells. Such action has been observed, inter alia, in the case of MCF-7 and MDA-MB-231 breast cancer cells [23, 24], PC3 prostate cancer cell [25] Me45 melanoma cell [18], hepatocellular carcinoma (HCC) cells [26], AGS gastric cancer cell line [27] Ishikawa and KLE endometrial carcinoma cell lines [28]. No Nampt receptor has been identified so far and the stimulating effect of Nampt on proliferation most probably occurs through AKT/PI3K, ERK/ MAPK and GSK- $3 \beta$ proteins signaling pathways [23, $25,26,28]$.

The results of our research confirm these numerous reports about the stimulating effect of eNampt on the proliferation of cancer cells. In these studies we used various eNampt concentrations, including those similar to physiological values found in human and rat serum [37-40]. It should be noted that eNampt serum concentrations in various types of 
cancer are usually elevated $[21,22,28,37-39,41$ 43]. Another significant difference is the reaction of the studied cells to Fk866, which is a specific Nampt inhibitor [44, 45]. In the culture of H295R cells, Fk866 strongly inhibits proliferation, while in the primary culture of rat adrenocortical cells, this inhibitor has no influence on proliferation, at least in the period studied (48h). It is also surprising to note that in primary culture of rat adrenocortical cells Fk866 does not modify the effect of ACTH on the degree of proliferation of these cells. This observation may suggest that in vitro ACTH-induced inhibition of adrenocortical cell proliferation is not associated with increased energy demand.

In the light of the above results, it should be emphasized that the cultured rat adrenocortical cells retained the normal reaction to ACTH added. In culture of these cells ACTH strongly inhibits proliferation, which was also observed in the present study $[36,46,47]$. We could not perform a similar experiment on H295R cells. H295R cells do not respond to corticotropin stimulation and do not exhibit MC2R (ACTH receptor) expression at both mRNA and protein levels $[48,49]$.

The differences observed in the proliferation of H295R cells and rat adrenocortical cells exposed to eNampt and/or Fk866 are most probably related to the "energy state" of these cells. Nampt is essential for the biosynthesis of NAD and found to be upregulated in many cancer cells. In addition, previous studies indicate that NAD formed as a result of Nampt activity is required to control the proliferative activity of different cells $[11,12,50]$. Cultured rat adrenocortical cells most likely maintain normal physiological levels of Nampt and under these conditions the probable inhibitory effect of Fk866 is less marked.

\section{Conclusions}

Summing up, the research carried out on human H295R cells and on primary culture of rat adrenocortical cells revealed significant differences in their reaction to eNamp. The strong eNampt stimulating effect on proliferative activity of H295R cells and the lack of effect on rat cells indicate an important role of Nampt in cancer cell biology.

Our previous studies have shown that iNampt have great impact on basal steroid hormone production of primary rat adrenal cells. The inhibition of iNampt by Fk866 leads to decrease of basal corticosterone and aldosterone production[33].

\section{Acknowledgements}

The present study was supported by PRELUDIUM (grant no. 2016/21/N/NZ4/00122) from the National Science Centre (Poland, Kraków)

\section{Author details}

Piotr Celichowski, Department of Histology and Embryology, Poznan University of Medical Sciences, ul Święcickiego 6, 60-781 Poznań, Poland, e-mail: p.celichowski@gmail.com

\section{Conflict of interest statement}

The authors state no conflict of interest

\section{References}

1. Revollo JR, Korner A, Mills KF, Satoh A, Wang T, Garten A, Dasgupta B, Sasaki Y, Wolberger C, Townsend RR, Milbrandt J, Kiess W, Imai S. Nampt/ $\mathrm{PBEF} /$ Visfatin regulates insulin secretion in beta cells as a systemic NAD biosynthetic enzyme. Cell Metab. 2007;6(5):363-75; DOI:10.1016/j. cmet.2007.09.003.

2. Bogan KL, Brenner C. Nicotinic acid, nicotinamide, and nicotinamide riboside: a molecular evaluation of NAD+ precursor vitamins in human nutrition. Annu Rev Nutr. 2008;28:115-30; DOI:10.1146/annurev. nutr.28.061807.155443.

3. Houtkooper RH, Canto C, Wanders RJ, Auwerx J. The secret life of NAD+ an old metabolite controlling new metabolic signaling pathways. Endocr Rev. 2010; 31(2):194-223; DOI:10.1210/er.2009-0026.

4. Borradaile NM, Pickering JG. NAD(+), sirtuins, and cardiovascular disease. Curr Pharm Des. 2009:15(1):110-7.

5. Fulco M, Cen Y, Zhao P, Hoffman EP, McBurney MW, Sauve AA, Sartorelli V. Glucose restriction inhibits skeletal myoblast differentiation by activating SIRT1 through AMPK-mediated regulation of Nampt. Dev Cell. 2008;14(5):661-73; DOI:10.1016/j.devcel.2008.02.004.

6. Hsu CP, Oka S, Shao D, Hariharan N, Sadoshima J. Nicotinamide phosphoribosyltransferase regulates cell survival through NAD+ synthesis in cardiac myocytes. Circ Res. 2009; 105(5):481-91; D0I:10.1161/ CIRCRESAHA.109.203703.

7. Pillai JB, Isbatan A, Imai S, Gupta MP. Poly(ADP-ribose) polymerase-1-dependent cardiac myocyte cell death during heart failure is mediated by NAD+ depletion and reduced Sir2alpha deacetylase activity. J Biol Chem. 2005;280(52):43121-30; DOI:10.1074/jbc.M506162200.

8. Revollo JR, Grimm AA, Imai S. The NAD biosynthesis pathway mediated by nicotinamide phosphoribosyltransferase regulates Sir2 activity in mammalian cells. J Biol Chem. 2004;279(49):50754-63; DOI:10.1074/ jbc.M408388200.

9. Rongvaux A, Galli M, Denanglaire S, Van Gool F, Dreze PL, Szpirer C, Bureau F, Andris F, Leo O. Nicotinamide phosphoribosyl transferase/ pre-B cell colony-enhancing factor/visfatin is required for lymphocyte development and cellular resistance to genotoxic stress. J Immunol. 2008;181(7):4685-95.

10. Song EK, Lee YR, Yu HN, Kim UH, Rah SY, Park KH, Shim IK, Lee SJ, Park YM, Chung WG, Kim JS, Han MK. Extracellular NAD is a regulator for FcgammaR-mediated phagocytosis in murine macrophages. Biochem Biophys Res Commun. 2008;367(1):156-61; DOI:10.1016/j. bbrc.2007.12.131.

11. Chen H, Wang S, Zhang H, Nice EC, Huang C. Nicotinamide phosphoribosyltransferase (Nampt) in carcinogenesis: new clinical opportunities. Expert Rev Anticancer Ther. 2016;16(8):827-38; DOI:10.1080/147371 40.2016.1190649.

12. Chiarugi A, Dolle C, Felici R, Ziegler M. The NAD metabolome--a key determinant of cancer cell biology. Nat Rev Cancer. 2012;12(11):741-52; DOI:10.1038/nrc3340

13. Shackelford RE, Mayhall K, Maxwell NM, Kandil E, Coppola D. Nicotinamide phosphoribosyltransferase in malignancy: a review. Genes Cancer. 2013;4(11-12):447-56; DOI:10.1177/1947601913507576.

14. Nergiz Avcioglu S, Altinkaya SO, Kucuk M, Yuksel H, Omurlu IK, Yanik S. Visfatin concentrations in patients with endometrial cancer. Gynecol Endocrinol. 2015;31(3):202-7; DOI:10.3109/09513590.2014.975687.

15. Zhao Y, Hu Q, Cheng F, Su N, Wang A, Zou Y, Hu H, Chen X, Zhou HM, Huang X, Yang K, Zhu Q, Wang X, Yi J, Zhu L, Qian X, Chen L, Tang Y, Loscalzo J, and Yang Y, SoNar, a Highly Responsive NAD+/NADH Sensor, Allows High-Throughput Metabolic Screening of Anti-tumor Agents. Cell Metab. 2015;21(5):777-89; DOI:10.1016/j.cmet.2015.04.009.

16. Bi TQ Che XM. Nampt/PBEF/visfatin and cancer. Cancer Biol Ther 2010;10(2):119-25; DOI:10.4161/cbt.10.2.12581.

17. Shackelford RE, Bui MM, Coppola D, Hakam A. Over-expression of nicotinamide phosphoribosyltransferase in ovarian cancers. Int J Clin Exp Pathol. 2010;3(5):522-7.

18. Buldak RJ, Buldak L, Polaniak R, Kukla M, Birkner E, Kubina R, Kabala-Dzik A, Dulawa-Buldak A, Zwirska-Korczala K. Visfatin affects redox adaptative responses and proliferation in Me45 human malignant melanoma cells: an in vitro study. Oncol Rep. 2013;29(2):771-8; DOI:10.3892/or.2012.2175.

19. Olesen UH, Petersen JG, Garten A, Kiess W, Yoshino J, Imai S, Christensen MK, Fristrup P, Thougaard AV, Bjorkling F, Jensen PB, Nielsen SJ, Sehested $M$. Target enzyme mutations are the molecular basis for resistance towards pharmacological inhibition of nicotinamide phosphoribosyltransferase. BMC Cancer. 2010;10:677; DOI:10.1186/1471-2407-10-677. 
20. Guo J, Lam LT, Longenecker KL, Bui MH, Idler KB, Glaser KB, Wilsbacher JL, Tse C, Pappano WN, Huang TH. Identification of novel resistance mechanisms to NAMPT inhibition via the de novo NAD(+) biosynthesis pathway and NAMPT mutation. Biochem Biophys Res Commun. 2017;491(3):681-686; DOI:10.1016/j.bbrc.2017.07.143.

21. Carbone F, Liberale L, Bonaventura A, Vecchie A, Casula M, Cea M, Monacelli F, Caffa I, Bruzzone S, Montecucco F, Nencioni A. Regulation and Function of Extracellular Nicotinamide Phosphoribosyltransferase/Visfatin. Compr Physiol. 2017;7(2):603-621; DOI:10.1002/cphy.c160029.

22. Garten A, Schuster S, Penke M, Gorski T, de Giorgis T, Kiess W. Physiological and pathophysiological roles of NAMPT and NAD metabolism. Nat Rev Endocrinol. 2015;11(9):535-46; DOI:10.1038/nrendo.2015.117.

23. Gholinejad Z, Kheiripour N, Nourbakhsh M, Ilbeigi D, Behroozfar K, Hesari Z, Golestani A, Shabani M, Einollahi N. Extracellular NAMPT/ Visfatin induces proliferation through ERK1/2 and AKT and inhibits apoptosis in breast cancer cells. Peptides. 2017;92:9-15; DOI:10.1016/j. peptides.2017.04.007.

24. Behrouzfar K, Alaee M, Nourbakhsh M, Gholinejad Z, Golestani A. Extracellular NAMPT/visfatin causes p53 deacetylation via NAD production and SIRT1 activation in breast cancer cells. Cell Biochem Funct. 2017;35(6):327-333; DOI:10.1002/cbf.3279.

25. Patel ST, Mistry T, Brown JE, Digby JE, Adya R, Desai KM, Randeva HS. A novel role for the adipokine visfatin/pre-B cell colony-enhancing factor 1 in prostate carcinogenesis. Peptides. 2010;31(1):51-7, DOI:10.1016/j. peptides.2009.10.001

26. Ninomiya S, Shimizu M, Imai K, Takai K, Shiraki M, Hara T, Tsurumi H, Ishizaki S, Moriwaki H. Possible role of visfatin in hepatoma progression and the effects of branched-chain amino acids on visfatin-induced proliferation in human hepatoma cells. Cancer Prev Res (Phila). 2011;4(12):2092-100; DOI:10.1158/1940-6207.CAPR-11-0340.

27. Mohammadi M, Zarghami N, Hedayati M, Ghaemmaghami S, Yamchi RM, Mohaddes M. Visfatin effects on telomerase gene expression in AGS gastric cancer cell line. Indian J Cancer. 2015;52(1):32-5 DOI:10.4103/0019-509X.175567

28. Wang G, Tian W, Liu Y, Ju Y, Shen Y, Zhao S, Zhang B, Li Y. Visfatin Triggers the Cell Motility of Non-Small Cell Lung Cancer via Up-Regulation of Matrix Metalloproteinases. Basic Clin Pharmacol Toxicol. 2016;119(6):548 554; DOI:10.1111/bcpt.12623.

29. Reverchon M, Rame C, Bunel A, Chen W, Froment P, Dupont J. VISFATIN (NAMPT) Improves In Vitro IGF1-Induced Steroidogenesis and IGF1 Receptor Signaling Through SIRT1 in Bovine Granulosa Cells. Biol Reprod. 2016;94(3):54; DOI:10.1095/biolreprod.115.134650

30. Diot M, Reverchon M, Rame C, Baumard Y, Dupont J. Expression and effect of NAMPT (visfatin) on progesterone secretion in hen granulosa cells. Reproduction. 2015;150(1):53-63; D0I:10.1530/REP-15-0021.

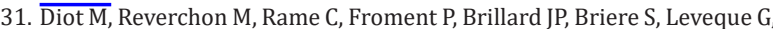
Guillaume D, Dupont J. Expression of adiponectin, chemerin and visfatin in plasma and different tissues during a laying season in turkeys. Reprod Biol Endocrinol. 2015;13:81; D0I:10.1186/s12958-015-0081-5.

32. Ocon-Grove OM, Krzysik-Walker SM, Maddineni SR, Hendricks GL 3rd Ramachandran R. NAMPT (visfatin) in the chicken testis: influence of sexual maturation on cellular localization, plasma levels and gene and protein expression. Reproduction. 2010;139(1):217-26; DOI:10.1530/ REP-08-0377

33. Celichowski P, Jopek K, Milecka P, Szyszka M, Tyczewska M, Malendowicz LK, Ruciński M. Nicotinamide phosphoribosyltransferase (Nampt) and the hypothalamic-pituitary-adrenal axis of the rat. Mol Med Rep. 2018;17(4):6163-6173; DOI:10.3892/mmr.2018.8569.

34. Trejter M, Hochol A, Tyczewska M, Ziolkowska A, Jopek K, Szyszka M, Malendowicz LK, Rucinski M. Visinin-like peptide 1 in adrenal gland of the rat. Gene expression and its hormonal control. Peptides. 2015;63:22-9; DOI:10.1016/j.peptides.2014.10.017.

35. Ziolkowska A, Rucinski M, Tyczewska M, Malendowicz LK. Orexin B inhibits proliferation and stimulates specialized function of cultured rat calvarial osteoblast-like cells. Int J Mol Med. 2008;22(6):749-55.

36. Rucinski M, Ziolkowska A, Szyszka M, Hochol A, Malendowicz LK. Evidence suggesting that ghrelin 0 -acyl transferase inhibitor acts at the hypothalamus to inhibit hypothalamo-pituitary-adrenocortical axis function in the rat. Peptides. 2012; 35(2):149-59; DOI:10.1016/j. peptides.2012.04.007.

37. Fazeli MS, Dashti H, Akbarzadeh S, Assadi M, Aminian A, Keramat MR, Nabipour I. Circulating levels of novel adipocytokines in patients with colorectal cancer. Cytokine. 2013;62(1):81-5; DOI:10.1016/j. cyto.2013.02.012

38. Nakajima TE, Yamada Y, Hamano T, Furuta K, Gotoda T, Katai H, Kato $\mathrm{K}$, Hamaguchi T, Shimada Y. Adipocytokine levels in gastric cancer patients: resistin and visfatin as biomarkers of gastric cancer. J Gastroenterol. 2009;44(7):685-90; DOI:10.1007/s00535-009-0063-5.
39. Yu-Duan T, Chao-Ping W, Chih-Yu C, Li-Wen L, Tsun-Mei L, Chia-Chang H, Fu-Mei C, Hsien-Chang L, Hsia-Fen H, Yau-Jiunn L, Jer-Yiing H. Elevated plasma level of visfatin/pre-b cell colony-enhancing factor in male oral squamous cell carcinoma patients. Med Oral Patol Oral Cir Bucal. 2013;18(2):e180-6.

40. Talavera-Urquijo E, Rodriguez-Navarro S, Beisani M, Salcedo-Allende MT, Chakkur A, Arus-Aviles M, Cremades M, Augustin S, Martell M, Balibrea JM. Morphofunctional Changes After Sleeve Gastrectomy and Very Low Calorie Diet in an Animal Model of Non-Alcoholic Fatty Liver Disease. Obes Surg. 2018;28(1):142-151; D0I:10.1007/s11695-017-2805-4.

41. Reddy PS, Umesh S, Thota B, Tandon A, Pandey P, Hegde AS, Balasubramaniam A, Chandramouli BA, Santosh V, Rao MR, Kondaiah P, Somasundaram K. PBEF1/NAmPRTase/Visfatin: a potential malignant astrocytoma/glioblastoma serum marker with prognostic value. Cancer Biol Ther. 2008;7(5):663-8.

42. Soncini D, Caffa I, Zoppoli G, Cea M, Cagnetta A, Passalacqua M, Mastracci L, Boero S, Montecucco F, Sociali G, Lasiglie D, Damonte P, Grozio A, Mannino E, Poggi A, D’Agostino VG, Monacelli F, Provenzani A, Odetti P, Ballestrero A, Bruzzone S, Nencioni A. Nicotinamide phosphoribosyltransferase promotes epithelial-to-mesenchymal transition as a soluble factor independent of its enzymatic activity. J Biol Chem. 2014;289(49):34189-204; DOI:10.1074/jbc.M114.594721.

43. Tian W, Zhu Y, Wang Y, Teng F, Zhang H, Liu G, Ma X, Sun D, Rohan T, Xue F. Visfatin, a potential biomarker and prognostic factor for endometrial cancer. Gynecol Oncol. 2013;129(3):505-12; DOI:10.1016/j. ygyno.2013.02.022.

44. Hasmann M, Schemainda I. FK866, a highly specific noncompetitive inhibitor of nicotinamide phosphoribosyltransferase, represents a novel mechanism for induction of tumor cell apoptosis. Cancer Res. 2003;63(21):7436-42.

45. Montecucco F, Cea M, Bauer I, Soncini D, Caffa I, Lasiglie D, Nahimana A Uccelli A, Bruzzone S, Nencioni A. Nicotinamide phosphoribosyltransferase (NAMPT) inhibitors as therapeutics: rationales, controversies, clinical experience. Curr Drug Targets. 2013;14(6):637-43.

46. Ramachandran J, Suyama AT. Inhibition of replication of normal adrenocortical cells in culture by adrenocorticotropin. Proc Natl Acad Sci U S A. 1975;72(1):113-7.

47. Rybak SM, Ramachandran J. Primary culture of normal rat adrenocortical cells. I. Culture conditions for optimal growth and function. In Vitro. 1981;17(7):599-604.

48. Parmar J, Key RE, Rainey WE. Development of an adrenocorticotropin-responsive human adrenocortical carcinoma cell line. J Clin Endocrinol Metab. 2008;93(11):4542-6; DOI:10.1210/jc.2008-0903.

49. Rainey WE, Saner K, Schimmer BP. Adrenocortical cell lines. Mol Cell Endocrinol. 2004;228(1-2):23-38; DOI:10.1016/j.mce.2003.12.020.

50. Cea M, Cagnetta A, Fulciniti M, Tai YT, Hideshima T, Chauhan D, Roccaro A, Sacco A, Calimeri T, Cottini F, Jakubikova J, Kong SY, Patrone F, Nencioni A, Gobbi M, Richardson P, Munshi N, Anderson KC. Targeting $\mathrm{NAD}+$ salvage pathway induces autophagy in multiple myeloma cells via mTORC1 and extracellular signal-regulated kinase (ERK1/2) inhibition. Blood. 2012;120(17):3519-29; DOI:10.1182/blood-2012-03-416776. 